\title{
Une utilisation simple du modèle HAYAMI en hydrologie
}

\author{
M. Baptista \\ C. Michel \\ CEMAGREF - Antony
}

\section{Introduction - le problème posé}

Il se pose souvent le problème de savoir comment une crue de projet est acheminée le long d'un tronçon de rivière. Il s'agit essentiellement d'avoir une idée sur l'atténuation de la pointe de crue et sur le temps d'arrivée de cette pointe. Or, il n'est pas toujours justifié de faire une étude hydraulique détaillée et cela pour deux raisons principales :

- les données hydrauliques complètes sont longues, délicates et coûteuses à obtenir : géométrie, caractéristiques de frottement, conditions aux limites;

- les données hydrologiques détaillées sont entachées d'incertitudes telles qu'il est souvent illusoire d'adjoindre un calcul hydraulique comparativement bien trop précis. En général, on connaît assez mal les apports latéraux, même si ceux-ci sont faibles pour le type de bief auquel on s'intéresse. De même, la forme de la crue de projet est assez incertaine et il faut souvent se contenter d'une description analytique simple dépendant de quelques paramètres.

Le but de ce papier est de fournir un outil simple pour répondre à ce genre de question. On va pour cela résumer une crue par deux paramètres quantitatifs : le débit maximum $Q$ et la durée $d$ pendant laquelle le débit dépasse $80 \%$ du débit maximum (fig. 1).
Le modèle d'Hayami nous permet une description très condensée d'un tronçon de rivière, car il ne fait intervenir que deux paramètres : la célérité $C$ et la diffusion $D$, en plus de la longueur du tronçon $L$. Il apparaît ainsi très adapté à la résolution du type de problème que nous nous posons.

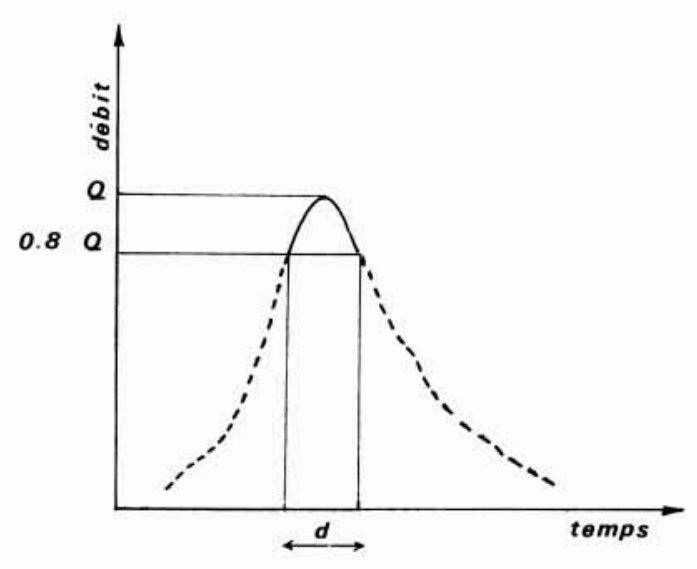

1. Paramètres $Q$ et d d'une crue.

\section{Simple using of HAYAMI's model in hydrology}

The present paper deals with the frequent problem of flood routing along a river reach. Our ain was to provide a simple tool suitable to give a quick and comprehensive answer. Quick, because the result is given simply by reading an abacus and comprehensive because this abacus is also used to identify the parameters specific to the reach under study. The model which is referred to is the diffusion model with constant coefficients, known in France as the Hayami model. When the flooding area is not too variable, the provided tool ought to be useful for a preliminary study. 


\section{Rappel du modèle HAYAMI}

Le modèle d'Hayami consiste à décrire la dynamique de l'écoulement en rivière par l'équation de l'onde de crue diffusante à paramètres constants.

Si $q$ est le débit, $t$ le temps et $x$ l'abscisse, cette équation s'écrit, en l'absence d'apports latéraux :

$$
\frac{\partial q}{\partial t}+C \frac{\partial q}{\partial x}-D \frac{\partial^{2} q}{\partial x^{2}}=0
$$

En considérant que le régime initial est un régime permanent (débit uniforme $q_{0}$ ), on démontre que l'on a :

$$
q(t, x)=q_{0}+\int_{0}^{t} q(\tau, 0) k(t-\tau, x) \mathrm{d} \tau
$$

avec

$$
k(\tau, x)=\frac{x}{2 \sqrt{\pi D} \tau^{3 / 2} \exp \left[(x-C \tau)^{2} / 4 D \tau\right]} .
$$

\section{La crue de projet : Une crue d'HAYAMI}

\subsection{Choix d'une forme de crue: «Crue d'Hayami pour un bief donné »}

Comme nous ne sommes pas très exigeants sur la forme précise de la crue de projet, dans la mesure où elle respecterait les caractéristiques essentielles $Q$ et $d$, on peut mettre à profit cette liberté pour choisir une forme analytique qui simplifie les calculs d'intégration impliqués par le modèle d'Hayami. Si l'hydrogramme, à l'entrée du tronçon de rivière considéré, a la forme du noyau d'Hayami (3), le résultat de l'intégration est immédiat. En effet, si l'hydrogramme à l'amont est donné par l'expression :

$$
\begin{aligned}
q(t)= & \frac{\sigma}{2 \sqrt{\pi \theta}(t+\beta)^{3 / 2} \exp \left\{\left[t^{2}-2 t(\alpha+\beta)+\right.\right.} \\
& \left.\left.+(\alpha+\beta)^{2}\right] /\left[4 \theta^{2}(t+\beta)\right]\right\}
\end{aligned}
$$

que nous pouvons noter $q(t, \alpha, \beta, \sigma, \theta)$, avec :

$-\alpha, \beta$ et $\sigma$ paramètres à caler sur l'hydrogramme réel ; - $\theta$ paramètre imposé en fonction du bief auquel on s'intéresse, $\theta=D / C^{2}$.

On peut montrer (MICHEL, 1982) que l'hydrogramme à la sortie d'un tronçon de longueur $L$, de paramètres $C$ et $D$ est donné directement par l'expression:

$$
q^{\prime}(t, \alpha+L / C, \beta, \sigma, \theta) .
$$

Il reste à caler les deux paramètres essentiels, que sont $Q$ et $d$, sur cet hydrogramme de projet, que nous désignerons désormais sous le nom de "crue d'Hayami pour un bief donné ", (fig. 2).

Dans ce qui suit on utilisera, pour simplifier, la dénomination plus ramassée de «crue d'Hayami ». Mais il faut se rappeler que cette crue est adaptée, au moyen d'un paramètre $(\theta)$, au bief que l'on traite.

\subsection{Débit de pointe d'une crue d'Hayami}

Etant donné une crue d'Hayami, représentée par l'expression (4) nous allons rechercher la valeur du débit maximum. Pour ce faire, il faut chercher la valeur de $t$ qui annule la dérivée de cette expression. Quelques calculs montrent que ce maximum $Q$ s'obtient pour une valeur de $t$ égale à $T$, donnée par :

$$
A=\frac{1}{B+\sqrt{1+B^{2}}}
$$

qui s'écrit également :

$$
B=1 / 2(1 / A-A)
$$

expressions dans lesquelles :

$$
A=T / \alpha
$$

et

$$
B=3 D / C^{2} \alpha .
$$

On montre alors que le débit maximum, obtenu pour $t=T$, vaut :

$$
Q=\frac{C^{2} \sigma}{D \alpha} G(B)
$$

avec :

$$
\begin{aligned}
G(B)=1 / 2 \sqrt{B / 3 \pi} & \left(B+\sqrt{1+B^{2}}\right)^{3 / 2} \times \\
\times & \exp \left[-3 B / 2\left(1+\sqrt{1+B^{2}}\right)\right] .
\end{aligned}
$$

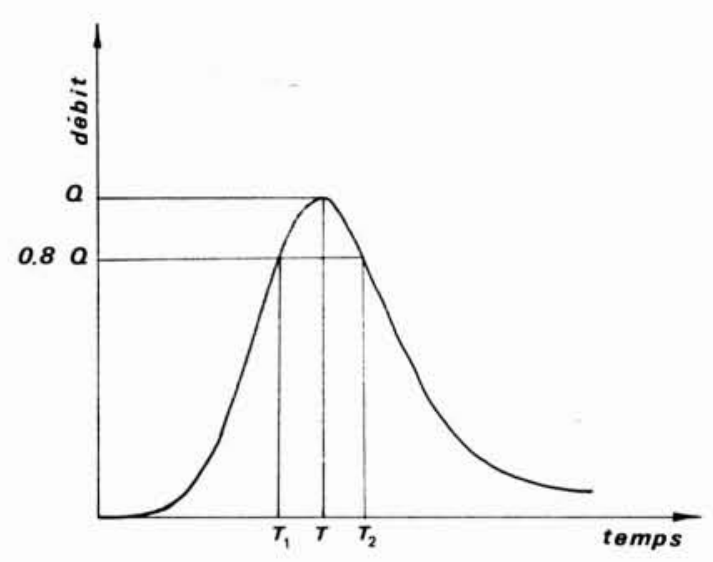

2. Une "crue d'HAYAMI" pour un bief donné. 


\subsection{Durée d'une crue d'Hayami}

Comme précisé dans le premier paragraphe, $d$ désigne la durée pendant laquelle le débit dépasse $80 \%$ du débit maximum $Q$. On cherche les deux racines, $T_{1}$ et $T_{2}$ (fig. 2) de l'équation en $t:$

c'est-à-dire :

$$
q(t, \alpha, \beta, \sigma, \theta)=0,8 Q
$$

$$
q(t, \alpha, \beta, \sigma, \theta)=0,8 q(T, \alpha, \beta, \sigma, \theta) .
$$

Utilisant l'expression (4), ainsi que la relation (6), et en notant $U=t / T$, on obtient l'équation en $U$ donnant les deux racines $U_{1}$ et $U_{2}$, que nous noterons :

$$
f(U)=0
$$

et qui est donnée par :

$$
\begin{aligned}
f(U)=\ln (U)+\frac{(1-U)}{\left(1-A^{2}\right)}(1 / U & \left.-A^{2}\right)+ \\
& +2 / 3[\ln (0,8)] .
\end{aligned}
$$

Les deux valeurs, $T_{1}$ et $T_{2}$, s'obtiennent numériquement en fonction de $A^{2}$ et on peut ainsi en déduire $d=T_{2}-T_{1}$. Nous désignons l'application $A^{2} \Rightarrow d$ par :

$$
d=T H\left(A^{2}\right) \text {. }
$$

\subsection{Routage d'une crue d'Hayami}

La situation est donc la suivante : on a un bief avec une longueur $L$, caractérisé par les paramètres hydrauliques $C$ et $D$. Une pointe de crue, caractérisée par le débit maximum $Q$ et la durée $d$ se présente en amont du tronçon. Le problème est d'obtenir la crue à l'aval du tronçon, caractérisée par les éléments suivants (fig. 3) :

- le débit maximum $Q^{\prime}$;

- la durée $d^{\prime}$;

- le retard de la pointe $\Delta t$.
La solution proposée est de considérer que la crue dont il faut calculer le routage peut être décrite par l'expression (4), qui dépend de 4 paramètres et de l'origine des temps $t$, que nous remplaçons par $T$, date de l'obtention de la pointe de la crue à l'entrée du bief.

Les valeurs de $A$ et $B$ (expressions (7) et (8)) peuvent être déterminées à partir des développements précédents. En effet, avec les équations ( $\left.6^{\prime}\right)$ et (14), on obtient l'équation suivante en $A^{2}$ :

$$
\frac{2 A^{2} H\left[A^{2}\right]}{1-A^{2}}=\delta
$$

où $\delta$ représente : $C^{2} d / 3 D$.

D'après l'équation (7), et en remarquant que $y^{\prime}=$ $y+L$ on en déduit, après avoir noté $\Omega=C L / 3 D$ :

$$
\frac{1}{B^{\prime}}=\frac{1}{B}+\Omega
$$

ce qui nous donne l'atténuation :

$$
\frac{Q^{\prime}}{Q}=\frac{G\left(B^{\prime}\right)}{G(B)}
$$

Connaissant $B^{\prime}$, on en déduit $A^{\prime}$ par les relations (6) et (7)

En utilisant la relation (8), et en notant $\Delta t$ la quantité $T^{\prime}-T$ on peut montrer que l'on obtient :

$$
\frac{\Delta t}{d}=\frac{1}{\delta}\left(A^{\prime} / B^{\prime}-A / B\right) .
$$

En ce qui concerne la durée de la crue à l'aval, par application des relations (14), (7) et (8) et en remarquant que $T^{\prime}=T+\Delta t$, on obtient :

$$
\frac{d^{\prime}}{d}=\frac{A^{\prime}}{\delta B^{\prime}} H\left(A^{\prime 2}\right)
$$

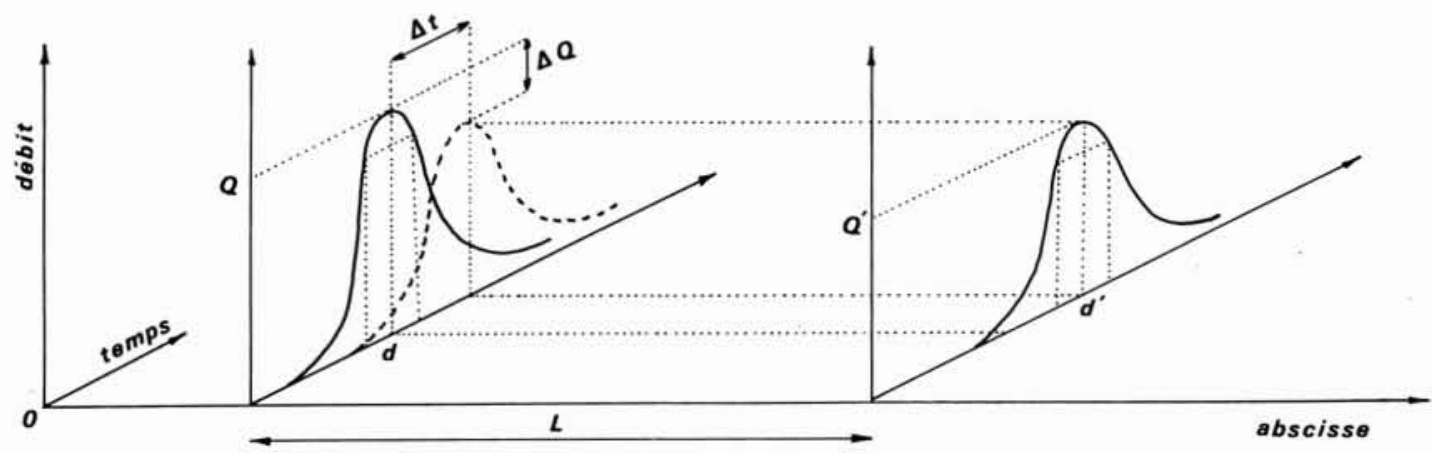

3. Représentation de la propagation de la crue. 


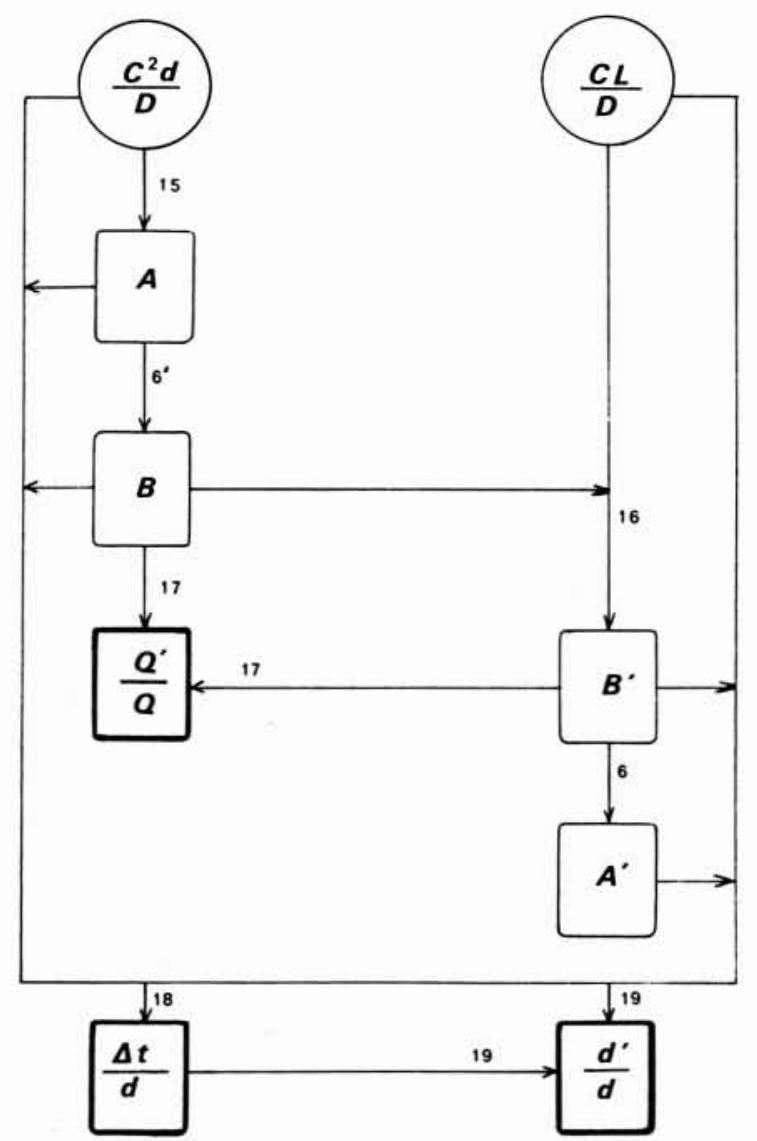

4. Schéma de la procédure de calcul.

Toute cette procédure peut être résumée dans le diagramme présenté à la figure 4 :

La résolution, une fois pour toutes de ces calculs, à partir d'un ensemble assez large de valeurs de $C^{2} d / D$ et $C L / D$ permet d'établir des abaques rendant le calcul du routage immédiat.

Pour des raisons de lisibilité de ces abaques, les valeurs calculées en sortie ont été légèrement transformées et on peut aisément en déduire l'atténuation et le décalage (abaque 1) ainsi que la durée $d^{\prime}$ de la crue à l'aval du bief (abaque 2).

\subsection{L'identification des paramètres $C$ et $D$}

Le tracé de l'abaque 1, compte tenu des variables choisies, permet de résoudre, ipso-facto, le problème inverse qui est l'identification des paramètres $C$ et $D$, ou leurs transformées $D / C$ et $D / C^{2}$.

En effet, pour une crue observée à l'amont $(Q, d)$ dont on connaît la résultante à l'aval d'un tronçon de longueur $L$, c'est-à-dire le décalage des pointes $\Delta t$ et l'atténuation $Q^{\prime} / Q$, on peut calculer $\ln \left[Q^{\prime} /\left(Q-Q^{\prime}\right)\right]$, ainsi que $\ln \left[(\Delta t / d) Q^{\prime} /\left(Q-Q^{\prime}\right)\right]$, ce qui permet de définir un point du graphique de l'abaque 1 . Les valeurs de $\ln \left(C^{2} d / D\right)$ et $\ln (C L / D)$ se lisent sur les cotes des courbes qui se croisent en ce point, ce qui permet d'obtenir $D / C^{2}$ et $D / C$ et, par suite, $C$ et $D$. Il faut noter que l'abaque fait apparaître un domaine où cette identification est impossible, qui est le cas où $\Delta t / d<$ $0,8\left(Q-Q^{\prime}\right) / Q^{\prime}$.

Cette identification des paramètres $C$ et $D$ est fondamentale, car les estimations a priori de ces paramètres en fonction des caractéristiques hydrauliques habituelles ne sont pas très fiables.

$\mathrm{Si}$ plusieurs crues observées sont disponibles, on pourra soit en déduire des valeurs moyennes (en prenant la moyenne géométrique), soit tenter d'associer les couples obtenus à certaines caractéristiques des crues (ampleur, débordement, ...). 

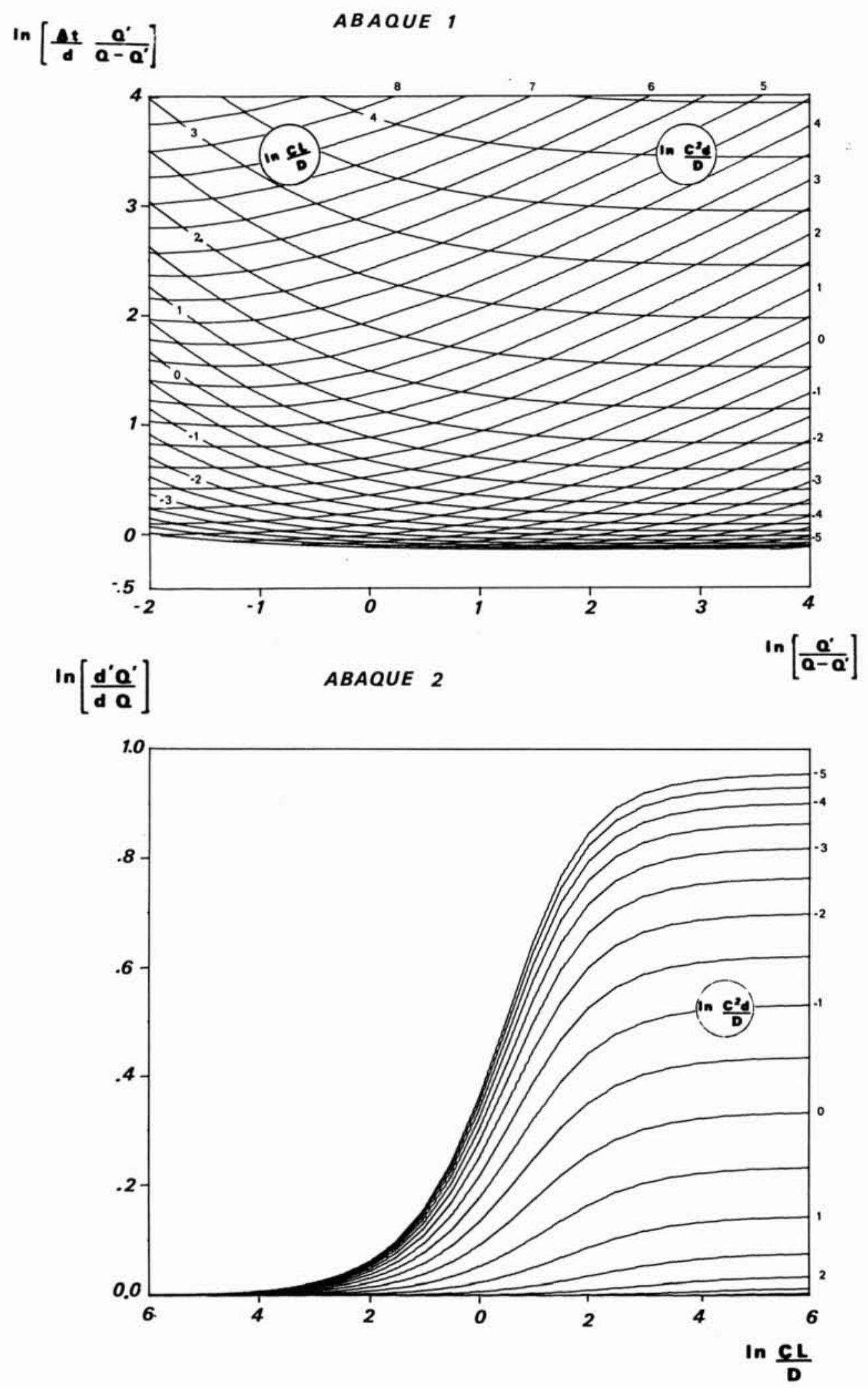
Tableau 1 : Caractéristiques des crues étudiées

\begin{tabular}{|c|c|c|c|c|c|c|c|}
\hline \multirow[t]{2}{*}{ Cours d'eau } & \multirow[t]{2}{*}{ Bief } & \multirow{2}{*}{$\begin{array}{l}\text { Longueur } \\
(\mathrm{km})\end{array}$} & \multirow[t]{2}{*}{ Date } & \multicolumn{2}{|c|}{ Crue Amont } & \multicolumn{2}{|c|}{ Crue Aval } \\
\hline & & & & $\begin{array}{l}\text { Débit } \\
\text { (m3/s) }\end{array}$ & $\begin{array}{l}\text { Durée } \\
\text { (h) }\end{array}$ & $\begin{array}{l}\text { Débit } \\
(\mathrm{m} 3 / \mathrm{s})\end{array}$ & $\begin{array}{c}\text { Retard } \\
\text { (h) }\end{array}$ \\
\hline La Seine & Troyes-Méry & 28,00 & $23 / 03 / 58$ & 193 & 96,00 & 182 & 38,40 \\
\hline La Seine & Bar-Méry & 70,00 & $14 / 01 / 55$ & 320 & 80,24 & 280 & 53,28 \\
\hline Wye River & Erwood-Belmont & 69,75 & $17 / 01 / 69$ & 370 & 11,62 & 310 & 13,89 \\
\hline Wye River & Erwood-Belmont & 69 , & $08 / 12 / 60$ & 1210 & 10,71 & 965 & 20,31 \\
\hline Wye River & Erwood-Belmont & 69,75 & $10 / 01 / 48$ & 700 & 9,60 & 445 & 20,56 \\
\hline Wye River & Erwood-Belmont & 69,75 & $26 / 11 / 39$ & 810 & 9,47 & 535 & 20,58 \\
\hline Wye River & Belmont-Redbrook & 80,50 & $04 / 12 / 60$ & 970 & 10,15 & 750 & 19,92 \\
\hline Wye River & Belmont-Redbrook & 80,50 & $09 / 12 / 65$ & 610 & 16,42 & 520 & 23,37 \\
\hline Rio Jacui & Itauba-Volta Grande & 36,00 & $17 / 12 / 81$ & 575 & 1,17 & 400 & 3,76 \\
\hline Rio Jacui & Itauba-Volta Grande & 36,00 & $18 / 12 / 81$ & 564 & 1.66 & 421 & 3,81 \\
\hline Rio Jacui & Itauba-Volta Grande & 36,00 & $28 / 12 / 81$ & 428 & 1,46 & 298 & 3,81 \\
\hline Rio Jacui & Itauba-Volta Grande & 36,00 & $29 / 12 / 81$ & 321 & 1,71 & 280 & 4,76 \\
\hline Rio Jacui & Itauba-Volta Grande & 36,00 & $02 / 05 / 81$ & 354 & 2,00 & 295 & 4,05 \\
\hline Rio Jacui & Itauba-Volta Grande & 36,00 & $23 / 05 / 81$ & 621 & 1.56 & 452 & 3,81 \\
\hline Rio Jacui & Itauba-Volta Grande & 36,00 & $25 / 03 / 82$ & 505 & 1,61 & 401 & 3,90 \\
\hline
\end{tabular}

Tableau 2 : Valeurs de célérité et diffusion

\begin{tabular}{llccc}
\hline Cours d'eau & Bief & Date & $\begin{array}{c}\text { Celerité } \\
\text { (m/s) }\end{array}$ & $\begin{array}{c}\text { Diffusion } \\
\text { (m2/s) }\end{array}$ \\
\hline La Seine & Troyes-Méry & $23 / 03 / 58$ & 0,20 & 1274 \\
La Seine & Bar-Méry & $14 / 01 / 55$ & 0,36 & 5271 \\
Wye River & Erwood-Belmont & $17 / 01 / 69$ & 1,39 & 8434 \\
Wye River & Erwood-Belmont & $08 / 12 / 60$ & 0,95 & 3041 \\
Wye River & Erwood-Belmont & $10 / 01 / 48$ & 0,92 & 6836 \\
Wye River & Erwood-Belmont & $26 / 11 / 39$ & 0,92 & 5541 \\
Wye River & Belmont-Redbrook & $04 / 12 / 60$ & 1,12 & 4537 \\
Wye River & Belmont-Redbrook & $09 / 12 / 65$ & 0,96 & 4188 \\
Rio Jacui & Itauba-Volta Grande & $17 / 12 / 81$ & 2,66 & 3294 \\
Rio Jacui & Itauba-Volta Grande & $18 / 12 / 81$ & 2,64 & 4273 \\
Rio Jacui & Itauba-Volta Grande & $28 / 12 / 81$ & 2,61 & 4359 \\
Rio Jacui & Itauba-Volta Grande & $29 / 12 / 81$ & 2,10 & 854 \\
Rio Jacui & Itauba-Volta Grande & $02 / 05 / 81$ & 2,46 & 2697 \\
Rio Jacui & Itauba-Volta Grande & $23 / 05 / 81$ & 2,64 & 4230 \\
Rio Jacui & Itauba-Volta Grande & $25 / 03 / 82$ & 2,56 & 2618 \\
\hline
\end{tabular}

Tableau 3 : Valeurs d'atténuation et de retard de la pointe

\begin{tabular}{|c|c|c|c|c|c|c|c|c|}
\hline \multirow[t]{2}{*}{ Cours d'eau } & \multirow{2}{*}{ Bief } & \multirow[t]{2}{*}{ Date } & \multicolumn{3}{|c|}{ Atténuation } & \multirow{2}{*}{$\begin{array}{c}\text { Retard } \\
\text { Obs. } \\
\text { (h) }\end{array}$} & \multirow{2}{*}{$\frac{\text { de la }}{\text { Calc. }}$} & \multirow{2}{*}{$\begin{array}{c}\text { Pointe } \\
\text { Erreur } \\
(\%)\end{array}$} \\
\hline & & & $\begin{array}{l}\text { Obs. } \\
(\%)\end{array}$ & $\begin{array}{c}\text { Calc. } \\
(\%)\end{array}$ & $\begin{array}{c}\text { Erreur } \\
(\%)\end{array}$ & & & \\
\hline La Seine & Troyes-Méry & $23 / 03 / 58$ & 5.70 & 4,11 & 27.8 & 38,40 & 21,41 & 44,2 \\
\hline La Seine & Bar-Méry & $14 / 01 / 55$ & 12,50 & 16,61 & 32,9 & 53,28 & 95,90 & 80,0 \\
\hline Wye River & Erwood-Belmont & $17 / 01 / 69$ & 16,22 & 25,20 & 55,4 & 13,89 & 20,65 & 48,7 \\
\hline Wye River & Erwood-Belmont & $08 / 12 / 60$ & 20,25 & 26,75 & 32,1 & 20,31 & 18,12 & 10,8 \\
\hline Wye River & Erwood-Belmont & $10 / 01 / 48$ & 36,43 & 25,93 & 28,8 & 20,56 & 18,02 & 12,4 \\
\hline Wye River & Erwood-Belmont & $26 / 11 / 39$ & 33,95 & 27,35 & 19,4 & 20,58 & 17,99 & 12,6 \\
\hline Wye River & Belmont-Redbrook & $04 / 12 / 60$ & 22,68 & 28,33 & 24,9 & 19,92 & 23,16 & 16.3 \\
\hline Wye River & Belmont-Redbrook & $09 / 12 / 65$ & 14,75 & 11,04 & 25,1 & 23,37 & 20,03 & 14,3 \\
\hline Rio Jacui & Itauba-Volta Grande & $17 / 12 / 81$ & 32,43 & 33,20 & 2,4 & 3,76 & 4,00 & 6.4 \\
\hline Rio Jacui & Itauba-Volta Grande & $18 / 12 / 81$ & 25,35 & 21,04 & $4 \quad 17,1$ & 3,81 & 4,00 & 5,0 \\
\hline Rio Jacui & Itauba-Volta Grande & $28 / 12 / 81$ & 30,37 & 24,82 & 218,3 & 3,81 & 3,99 & 4,8 \\
\hline Rio Jacui & Itauba-Volta Grande & $29 / 12 / 81$ & 12,77 & 22,32 & 274,8 & 4,76 & 3,85 & $5 \quad 19,1$ \\
\hline Rio Jacui & Itauba-Volta Grande & $02 / 05 / 81$ & 16,67 & 16,55 & 0,7 & 4,05 & 3.96 & 2,3 \\
\hline Rio Jacui & Itauba-Volta Grande & $23 / 05 / 81$ & 27,21 & 22,91 & 15,8 & 3,81 & 4,00 & 5,0 \\
\hline Rio Jacui & Itauba-Volta Grande & $25 / 03 / 82$ & 20,59 & 22,90 & 11,2 & 3,90 & 3,98 & 2,1 \\
\hline ERREUR M & & & & & 25 & & & 18,9 \\
\hline
\end{tabular}




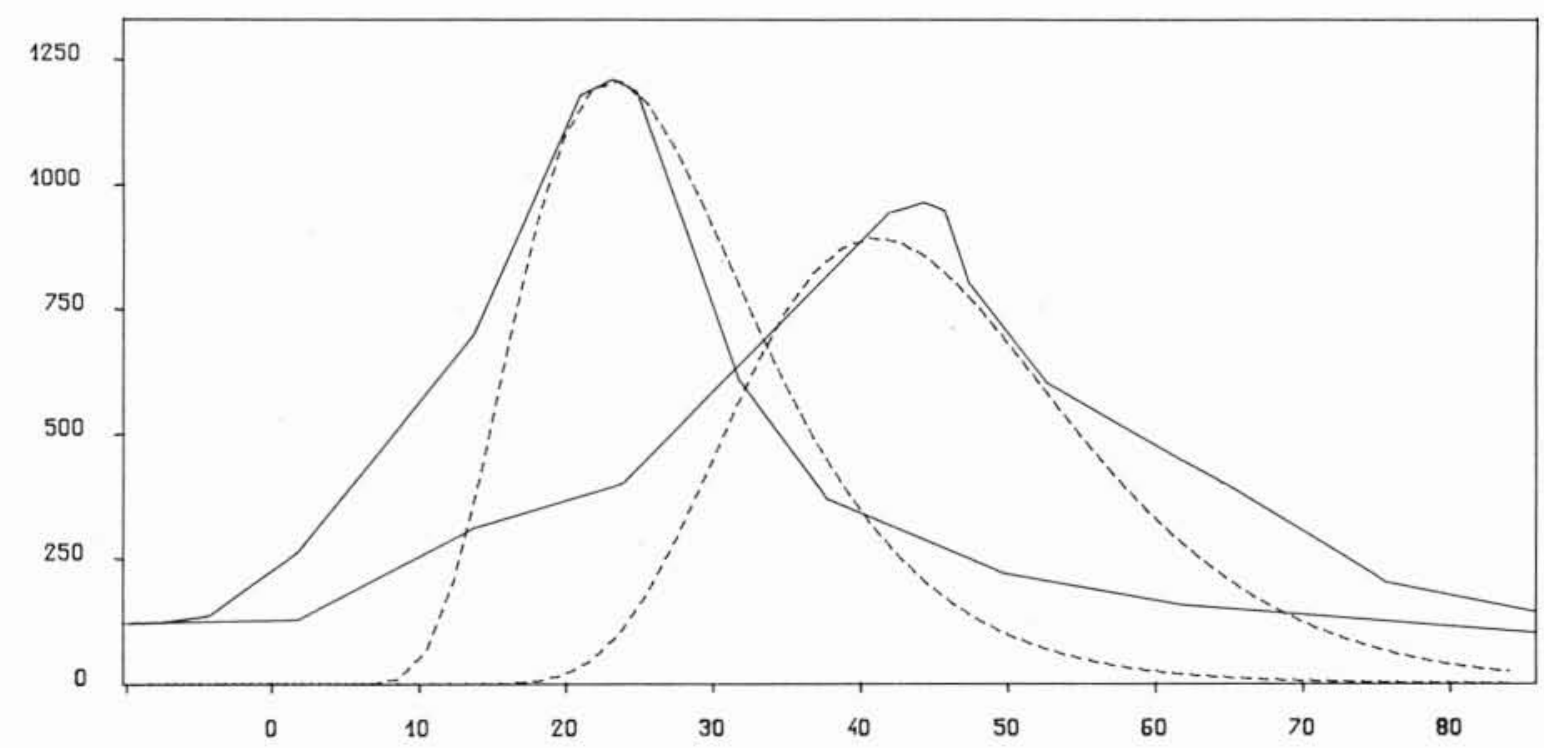

5. Hydrogrammes observés et calculés.

\section{Etude de cas réels}

Dans le but de vérifier l'applicabilité de la méthodologie de façon concrète, on a travaillé sur quelques cas, cités dans la littérature (I.P.H., 1983), (LEBRETON et BENOIST, 1968), (N.E.R.C., 1975) et (ZAMANILLO et TuCCI, 1987). Les caractéristiques des crues sur lesquelles on a travaillé sont présentées dans le tableau 1 .

Comme on l'a vu précédemment, le problème est d'abord d'identifier la Célérité et la Diffusion. En utilisant l'abaque 1, avec les données présentées dans le tableau 1 , on peut identifier ces paramètres pour chaque crue, comme présenté au tableau 2.

On peut maintenant tester la validité des valeurs de $C$ et $D$ pour l'évaluation de l'atténuation et du retard de la pointe de crue. Pour ce faire, conformément à ce qui serait fait dans la pratique, on a pris la moyenne géométrique des valeurs de $D / C$ et $D / C^{2}$ de toutes les crues du bief, sauf, bien sûr de la crue choisie comme test. Ainsi, pour l'étude de la crue de 1969 pour la Wye River, par exemple, on a utilisé les moyennes des valeurs calées d'après les crues de 1960, 1939 et 1948. L'abaque 1 nous donne alors les valeurs de $Q^{\prime} /\left(Q-Q^{\prime}\right)$ et de $\Delta t / d$, et donc l'atténuation et le retard de la pointe de la crue. Les valeurs ainsi calculées, de même que les valeurs des erreurs commises sont présentées au tableau 3.

L'erreur moyenne pour l'atténuation est de $25 \%$ environ. Sachant que les atténuations, dans notre échantillon, sont de l'ordre de $20 \%$, l'erreur commise, exprimée en pourcentage, de l'évaluation du débit aval est de $5 \%$ environ. Pour le retard de la pointe de crue, on peut voir que l'erreur moyenne est inférieure à $20 \%$.

Ces ordres de grandeur semblent être tout à fait compatibles avec les erreurs d'ordre hydrologique, et, peuvent être acceptables dans la pratique usuelle.
A titre d'illustration, on peut voir sur la figure 5 les hydrogrammes, observés et d'Hayami, amont et aval de la crue de décembre 1960 (Wye River, tronçon 1).

\section{Extensions possibles}

La méthodologie qu'on vient de proposer est assez souple pour nous permettre de faire face à une gamme importante de situations réelles, en permettant d'aborder des problèmes plus complexes, comme les crues multiples. On analyse dans les paragraphes qui suivent quelques généralisations possibles

En effet, on est souvent obligé de traiter des tronçons de cours d'eau qui sont constitués d'une succession de biefs homogènes ( $C$ et $D$ constants à l'intérieur de chaque bief). Dans ces conditions, on doit traiter séparément chaque bief, jugé homogène, en utilisant d'abord la démarche proposée sur le premier bief. On détermine alors les valeurs de $Q^{\prime}$, et $\Delta t$ (abaque 1) et de $d^{\prime}$ (abaque 2). On utilise ensuite ces caractéristiques de la crue à l'aval de ce bief comme données amont pour le deuxième bief, et, successivement jusqu'à la fin de tronçon étudié. On obtient ainsi le débit après routage et le retard de la pointe, constitué de la somme des retards relatifs à chaque bief.

Un autre problème qui se pose est l'occurrence de crues complexes, constituées, par exemple, de deux pointes. Du fait de la linéarité du modèle d'Hayami, cette situation pourrait être également traitée, en décomposant l'hydrogramme en amont du tronçon considéré en une somme de deux crues d'Hayami, qui peuvent être routées, séparément, selon la méthodologie proposée. 


\section{Conclusion}

La démarche proposée débouche sur une utilisation extrêmement simple du modèle d'Hayami. Cette simplicité s'accorde assez bien avec l'état des données disponibles dans la pratique quotidienne de l'hydrologie.

Il est parfois difficile d'obtenir des enregistrements continus qui soient fiables. En calant les paramètres $C$ et $D$ du modèle d'Hayami, sur les deux caractéristiques les plus pertinentes, on accroît nos chances d'atteindre l'objectif fixé.

\section{Bibliographie}

I.P.H. - Instituto de Pesquisas Hidraulicas, 1983: Estudos Hidrodinamicos do Rio Jacui - Rapport technique IPH/UFRS - Porto Alegre.

J. C. Lebreton, G. Benoist, 1968 : Influence du Réservoir Seine sur la propagation de crues de la Seine jusqu'à Paris. Xèmes journées de l'hydraulique, Question IV, Rapport 6, 8 p., S.H.F. - Paris.

C. MiCHEL, 1982: Utilisation de l'Onde de crue diffusante en hydrologie. Note Interne CEMAGREF, 9 p., Antony.

N.E.R.C. - Natural Environment Research Council, 1975: Flood Studies Report, volume III, Flood Routing Studies, London.

E. Zamanillo, C. E. Tucci, 1987 : Simulação de escoamento por Muskingun-Cunge com parametros variaveis. Actes du Congrès brésilien d'hydrologie et ressources en eau, p. 54 à 65 , vol. 3 , Salvador.

Adresse des auteurs :

Marcio Baptista, ingénieur, élève-chercheur de l'ENPC, en stage

à la Section hydrologie du CEMAGREF,

Claude Michel, IGREF à la Section hydrologie du CEMAGREF,

Groupement d'Antony

Division Hydrologie-Hydraulique,

BP 121

92164 Antony Cedex

Tel. : 40966121 


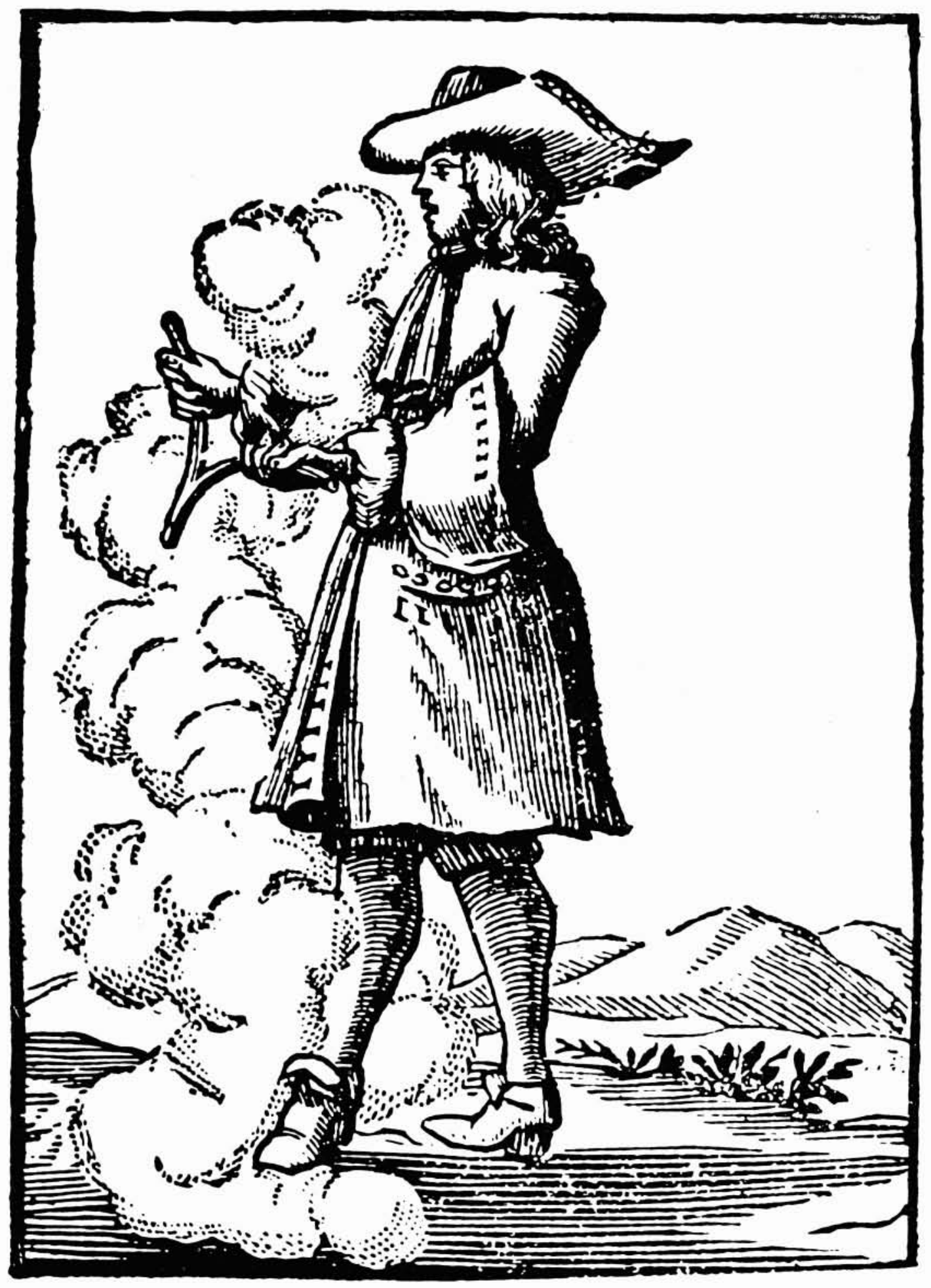




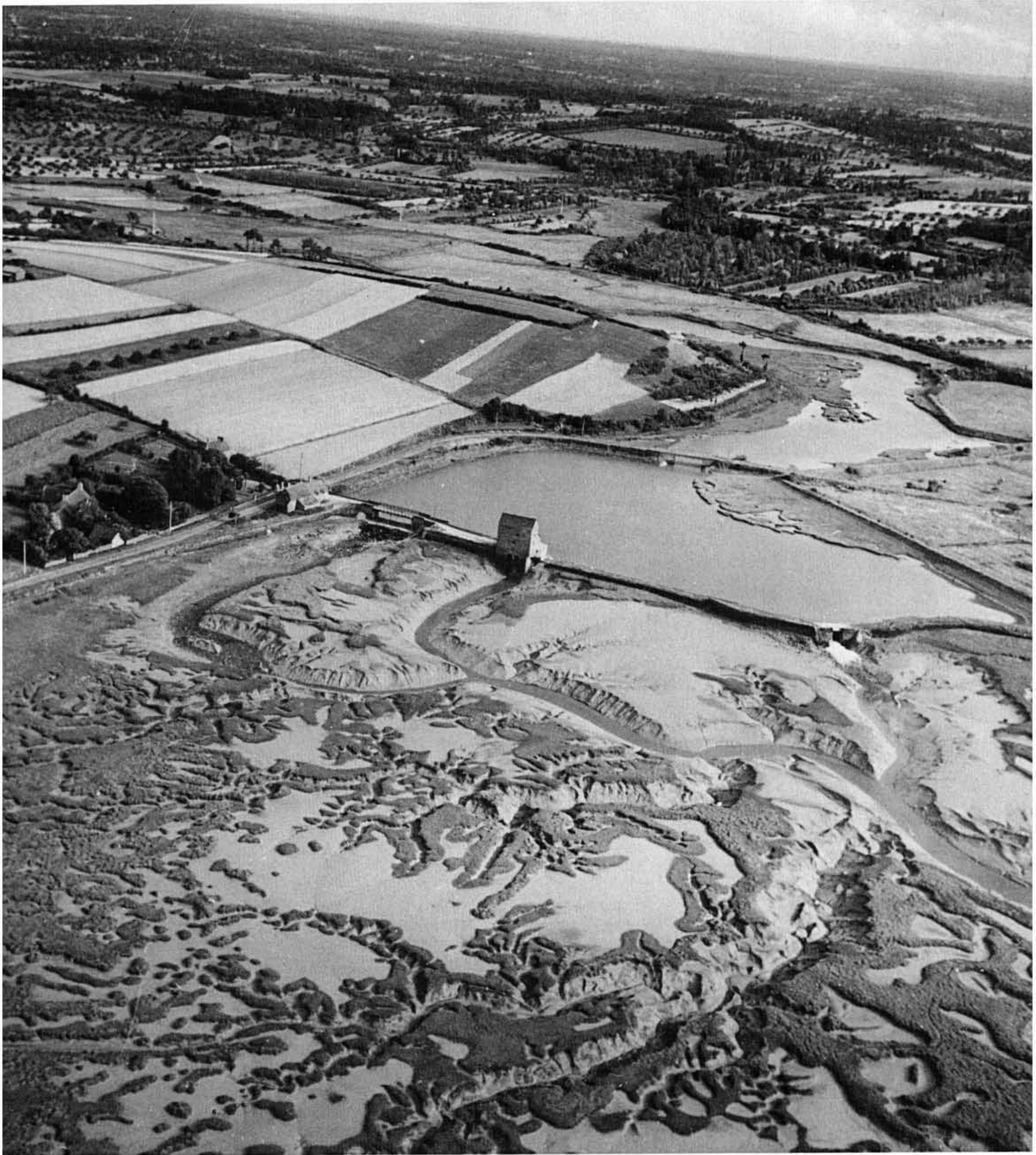

Moulin à marées sur la Rance. 